\title{
Diagnostic Accuracy of Five Different Fecal Markers for the Detection of Precancerous and Cancerous Lesions of the Colorectum
}

\author{
Mariann Rutka, ${ }^{1}$ Renáta Bor, ${ }^{1}$ Anita Bálint, ${ }^{1}$ Anna Fábián, ${ }^{1}$ Ágnes Milassin, ${ }^{1}$ Ferenc Nagy, \\ Zoltán Szepes, ${ }^{1}$ Mónika Szú́cs, ${ }^{2}$ László Tiszlavicz, ${ }^{3}$ Klaudia Farkas, ${ }^{1}$ and Tamás Molnár ${ }^{1}$ \\ ${ }^{1}$ First Department of Medicine, University of Szeged, Korányi Fasor 8-10, Szeged 6720, Hungary \\ ${ }^{2}$ Department of Medical Physics and Informatics, University of Szeged, Korányi Fasor 9, Szeged 6720, Hungary \\ ${ }^{3}$ Department of Pathology, University of Szeged, Állomás Utca 2, Szeged 6720, Hungary
}

Correspondence should be addressed to Mariann Rutka; rutka.mariann@gmail.com

Received 3 March 2016; Revised 15 May 2016; Accepted 19 May 2016

Academic Editor: Krishna Reddy

Copyright (C) 2016 Mariann Rutka et al. This is an open access article distributed under the Creative Commons Attribution License, which permits unrestricted use, distribution, and reproduction in any medium, provided the original work is properly cited.

\begin{abstract}
Background. Colorectal cancer (CRC) is the second deadliest malignancy worldwide. This study aimed to compare the diagnostic accuracy of different fecal markers in the detection of colorectal adenomas and cancer. Methods. Stool samples of patients referred to colonoscopy were collected for the analysis of tumor $\mathrm{M}_{2}$ pyruvate kinase $\left(\mathrm{M}_{2} \mathrm{PK}\right)$, human hemoglobin (Hb), hemoglobin/haptoglobin ( $\mathrm{Hb} / \mathrm{Hp})$ complex, fecal calprotectin (FC), and matrix metalloproteinase-9 (MMP-9). Results. Sensitivity and specificity of $\mathrm{M}_{2} \mathrm{PK}$ for adenomas sized $>1 \mathrm{~cm}$ were $60 \%$ and $67.5 \%$ and for CRC were $94.7 \%$ and $67.5 \%$. Sensitivity and specificity of iFOBT for adenomas sized $\geq 1 \mathrm{~cm}$ were $80 \%$ and $72.5 \%$ and for CRC were $94.7 \%$ and $72.5 \%$. Sensitivity and specificity of $\mathrm{Hb} / \mathrm{Hp}$ complex for adenomas sized $\geq 1 \mathrm{~cm}$ were $80 \%$ and $52.9 \%$ and for CRC were $100 \%$ and $52.9 \%$. Sensitivity of FC and MMP-9 for CRC was $77.8 \%$ and $72.2 \%$. Combined use of $\mathrm{M}_{2} \mathrm{PK}$, iFOBT, and FC resulted in a sensitivity and specificity of $95 \%$ and $47.5 \%$ for the detection of adenomas sized $\geq 1 \mathrm{~cm}$. Discussion. In CRC, sensitivity of $\mathrm{M}_{2} \mathrm{PK}$, iFOBT, and $\mathrm{Hb} / \mathrm{Hp}$ complex proved to be high. Combined use of $\mathrm{M}_{2} \mathrm{PK}$, iFOBT, and FC may be valuable in the detection of large adenomas.
\end{abstract}

\section{Introduction}

Colorectal cancer (CRC) incidence and mortality rates vary markedly worldwide. Globally, CRC is a third most common cancer, being a significant leading cause of cancer death in both genders [1]. Furthermore, the incidence of CRC is increasing in Central European countries [1]. The Hungarian mortality rates for CRC proved to be the highest among men in Europe in 2012 [2].

The vast majority of CRC cases are sporadic colon cancers characterized by a multistep carcinogenic process [3]. Advanced adenomas greater than $10 \mathrm{~mm}$ in diameter with high-grade dysplasia or with more than $20 \%$ villous component are considered to be the clinically relevant precursors of CRC. However, the long premalignant phase of sporadic CRCs provides a good opportunity for successful screening and intervention.
Colonoscopy is considered the gold standard of CRC screening tools. However, mainly due to the invasive nature of colonoscopy, the acceptance of this type of screening method among patients is low. The most commonly used noninvasive screening method for CRC is the guaiac fecal occult blood test (gFOBT) based on the detection of hemoglobin peroxidase activity in the stool. However, the sensitivity and the specificity of this test are not good enough to safely rule out the presence of CRC or adenomas which is why there is a great need for a better noninvasive marker for these conditions. In the case of proximal malignant lesions, hemoglobin/haptoglobin $(\mathrm{Hb} / \mathrm{Hp})$ detection can be superior to $\mathrm{Hb}$ detection alone since $\mathrm{Hb} / \mathrm{Hp}$ complex remains stable over the entire course of the large bowel in comparison to $\mathrm{Hb}$ degraded on the way [4-6]. $\mathrm{M}_{2}$ pyruvate kinase (PK) is a biochemical form of PK which is a key enzyme in cancer cell metabolism [7]. $\mathrm{M}_{2} \mathrm{PK}$ is expressed in normal 
proliferating cells, embryonic cells, adult stem cells, and cancer cells [8]. Elevated levels of $\mathrm{M}_{2} \mathrm{PK}$ have been detected in colonic adenocarcinoma [9]. Calprotectin is a calciumbinding and zinc-binding protein complex that is abundant in the cytosol of inflammatory cells $[10,11]$. Fecal calprotectin (FC), a biomarker of intestinal inflammation, has been in clinical use for years in inflammatory bowel disease [11-13]. FC has been shown to be elevated in CRC and has been suggested to be for screening high risk groups for CRC [14].

Matrix metalloproteinase (MMP) is a large family of calcium-dependent zinc-containing endopeptidases responsible for tissue remodelling and degradation of the extracellular matrix components, including collagens, elastins, gelatin, matrix glycoproteins, and proteoglycan, in multiple disease settings including malignant processes. MMP-9 subtypes are believed to play a crucial role in the progression and metastasis formation of many tumors, including CRC [15].

Since the majority of the abovementioned tests are not officially recommended in the CRC screening guidelines and some of them have not been tested previously, the aim of this study was to compare the diagnostic accuracy of different fecal markers in the detection of precancerous and cancerous lesions of the colorectum and to find the most accurate for CRC screening.

\section{Methods}

2.1. Patient Population and Study Protocol. Patients from the 1st Department of Medicine, University of Szeged, who were referred for colonoscopy were invited to participate in the study. Data on symptoms, smoking habits, family history, and current medication were collected. Every patient was informed about the study details and asked to sign written consent. The patients were instructed for sample collection and handling. All patients were asked to collect stool samples one day before administration of bowel preparation. Plastic containers were provided for feces collection. After bringing the samples at the lab of the clinic, they were frozen at $-20^{\circ} \mathrm{C}$ until further analysis. Patients did not have to keep a special diet and were told to take their usual medications. Selection of the patient groups with adenomas sized $<1 \mathrm{~cm}$ and $\geq 1 \mathrm{~cm}$ and CRC was based on the endoscopic and histological finding. The stool testing for $\mathrm{M}_{2} \mathrm{PK}$, iFOBT, FC, and MMP-9 was carried out by a single trained person who was blinded to the results of the colonoscopy.

The study was approved by the Regional and Institutional Human Medical Biological Research Ethics Committee of the University of Szeged.

2.2. Measurement of Fecal $M_{2} P K$ and iFOBT. A combined rapid immunochromatographic lateral flow test was used for simultaneous detection of enzyme biomarker $\mathrm{M}_{2} \mathrm{PK}$ and human hemoglobin (combined $\mathrm{M}_{2} \mathrm{PK}$ and $\mathrm{HB}, 2$ in 1 Quick Test, ScheBo ${ }^{\circledR}$ Biotech). For these measurements, stool samples were thawed and a special stick capturing $4 \mathrm{mg}$ of stool was loaded. These tests are based on visual inspection of colors at test and control lines. The result is exclusively qualitative (detection limit of $\mathrm{M}_{2} \mathrm{PK}$ was $4 \mathrm{U} / \mathrm{mL}$; detection limit of $\mathrm{Hb}$ was $15 \mathrm{ng} / \mathrm{mL}$ ).
2.3. Measurement of Fecal $\mathrm{Hb}$ and $\mathrm{Hb} / \mathrm{Hp}$ Complex. $\mathrm{Hb} / \mathrm{Hp}$ complex was determined from stool samples with a visual immunochromatographic quick test: ColonView $\mathrm{Hb}$ and $\mathrm{Hb} / \mathrm{Hp}$ fecal occult blood test (Biohit HealthCare; detection limit of $\mathrm{Hb}$ was $15 \mathrm{ng} / \mathrm{mL}$; detection limit of $\mathrm{Hb} / \mathrm{Hp}$ was $4 \mathrm{ng} / \mathrm{mL})$.

2.4. Measurement of FC and Fecal MMP-9. For FC measurements, fecal specimens were thawed at $4^{\circ} \mathrm{C}$. FC level was quantified by using enzyme-linked immunosorbent assay (Quantum Blue, BÜHLMANN Laboratories Ltd., Schönenbuch) according to the manufacturer's instructions. For MMP-9 measurements, $1 \mathrm{~g}$ of fecal samples was diluted, mixed, homogenised in $4 \mathrm{~mL}$ of ice-cold Tris-buffer $(0.15 \mathrm{M}$ $\mathrm{NaCl}+20 \mathrm{mM}$ Tris- $\mathrm{HCl}, \mathrm{pH} 8.3$ ), and then centrifuged. MMP-9 was also measured by quantitative enzyme-linked immunosorbent assay (R\&D Systems, Abingdon, UK) [16].

2.5. Colonoscopy and Histological Examination. Diagnosis was based on the endoscopic and histopathological findings. Colonoscopies were performed by three experienced endoscopists (TM, ZSZ, and FN) who were blinded to fecal tests results. Carcinomas were classified according to the Dukes staging system and location. Adenomatous polyps were classified according to histopathological characteristics, size (large polyps: $\geq 1 \mathrm{~cm}$; small polyps: $<1 \mathrm{~cm}$ ), and location. All colonoscopy biopsies were examined by an expert pathologist (LT). The diagnoses were reported using the standard WHO classification of colorectal neoplasia. In addition to their size, all polypoid lesions were classified as hyperplastic polyps or adenomas, being further classified according to their histological pattern as tubular, tubulovillous, villous, or serrate adenomas.

2.6. Statistical Analysis. CRCs and adenomas were analysed separately. The diagnostic value of fecal markers for detecting adenomas and CRCs was assessed by calculating the sensitivity and the specificity of the test. Correlations between FC and MMP-9 and endoscopic findings were determined by ANOVA method. The cut-off levels, specificity, and sensitivity between CRC and control groups were calculated using the receiver operating characteristic (ROC) analysis. All statistical analyses were carried out using STATA 9 (StataCorp, TX, 2005). $P$ values $<0.05$ were considered to be statistically significant.

\section{Results}

3.1. Patient Population. Ninety-five consecutive in- and outpatients admitted for total colonoscopy between September 2014 and April 2015 were prospectively enrolled in the study. Indications for colonoscopies were abdominal complaints, bloody stool, family history of CRC, and prior colorectal adenoma. Patients with active gastrointestinal bleeding, menstruation, and past history of total colectomy were excluded from the study. Study groups were defined on the basis of the result of colonoscopy and histological evaluation.

Mean age was 67 years (range: $21-92$ ) in study population. 57 female and 38 male patients were in these three groups, 
TABLE 1: Demographic characteristics of the study population.

\begin{tabular}{|c|c|c|c|c|}
\hline Demographic data & All patients $(95)$ & Control group (40) & Adenoma group (36) & Cancer group (19) \\
\hline Female/male & $38 / 57$ & $19 / 21$ & $14 / 22$ & $5 / 14$ \\
\hline Age (years) & $67(21-92)$ & $67(21-87)$ & $68(51-81)$ & $65(44-92)$ \\
\hline Current smokers & $13(13.7 \%)$ & $4(10 \%)$ & $5(13.9)$ & $4(21.1)$ \\
\hline \multicolumn{5}{|l|}{ Comorbidities } \\
\hline Hypertension & $54(56.8 \%)$ & $23(57.5 \%)$ & $22(61.1 \%)$ & $9(47.4 \%)$ \\
\hline Diabetes mellitus & $21(22.1 \%)$ & $7(17.5 \%)$ & $8(22.2 \%)$ & $6(31.6 \%)$ \\
\hline Hyperlipidaemia/hypercholesterinemia & $22(23.2 \%)$ & $9(22.5 \%)$ & $11(30.6 \%)$ & $2(10.5 \%)$ \\
\hline Cardiovascular disease & $25(26.3 \%)$ & $11(27.5 \%)$ & $10(27.7 \%)$ & $4(21.1 \%)$ \\
\hline Cerebrovascular disease & $13(13.7 \%)$ & $6(15 \%)$ & $4(11.2 \%)$ & $3(15.8 \%)$ \\
\hline Hyper/hypothyroidism & $13(13.7 \%)$ & $5(12.5 \%)$ & $7(19.4 \%)$ & $1(5.3 \%)$ \\
\hline Pulmonary disease & $6(6.3 \%)$ & $4(10 \%)$ & $2(5.6 \%)$ & 0 \\
\hline Gout & $11(11.6 \%)$ & $5(12.5 \%)$ & $5(13.9 \%)$ & $3(15.8 \%)$ \\
\hline Autoimmune disease & $4(4.2 \%)$ & 0 & $3(8.3 \%)$ & $1(5.3 \%)$ \\
\hline Malignant disease (simultaneously) & $3(3.2 \%)$ & $1(2.5 \%)$ & $2(5.6 \%)$ & 0 \\
\hline Hepatitis $(B, C)$ & $2(2.1 \%)$ & $1(2.5 \%)$ & $1(2.8 \%)$ & 0 \\
\hline Diverticulum & $24(25.3 \%)$ & $11(27.5 \%)$ & $10(27.8 \%)$ & $3(15.8 \%)$ \\
\hline Haemorrhoids & $20(21.1 \%)$ & $11(27.5 \%)$ & $7(19.4 \%)$ & $2(10.5 \%)$ \\
\hline
\end{tabular}

respectively. Demographic characteristics of the study population are summarized in Table 1 . Family history of CRC was reported by 26 patients. Considering therapy, 26 patients received aspirin or clopidogrel and 4 received acenocoumarol or heparin at the time of the investigation.

3.2. Colonoscopic and Histological Findings. Forty of the 95 patients included in the study represented the control group without any premalignant or malignant findings on endoscopy. Nine of the control patients presented with initial diverticulosis without any sign of inflammation. Colonoscopic findings in the remaining patients of the control group were totally normal.

Thirty-six patients were diagnosed with adenomas (adenoma group). In the adenoma group, 16 patients presented with adenomas sized $<1 \mathrm{~cm}$ and 20 with adenomas sized $\geq 1 \mathrm{~cm}$. Adenomas sized $<1 \mathrm{~cm}$ were equally located at the proximal and the distal part of the colon. The location of adenomas sized $\geq 1 \mathrm{~cm}$ in the majority (65\%) of the patients was the proximal part of the colon. In twenty-three adenomatous cases, a histologic sample was obtained. In the remaining thirteen cases, the samples were less than $1 \mathrm{~cm}$ and did not suggest the presence of malignancy. Based on the histological assessment of the samples $(n=23)$, in $78.3 \%$ of the cases (in 18 patients), the adenomas were with lowgrade dysplasia; in 13\% (in 3 patients), adenomas were with high-grade dysplasia; and in $8.7 \%$ (in 2 patients) there were hyperplastic polyps. In $56.5 \%$ of the patients the adenomas were of the tubular type, in $4.3 \%$ they were of the villous type, and in $30.4 \%$ they belong to the tubulovillous type.

Cancer was found in 19 cases, and, according to their histological evaluation, the tumors were identified as adenocarcinomas. In $89 \%$ of the patients, the cancer was located in the distal colon (in 10 patients in the rectum and in 7 patients in the sigmoid colon). In the remaining 2 cases, the tumor was located in the distal part of the transverse colon. $28.8 \%$ of
TABLE 2: The numbers of patients having different stages of cancer according to Dukes classification.

\begin{tabular}{lc}
\hline Dukes stage & Patients \\
\hline Carcinoma in situ & 1 \\
Dukes A & 3 \\
Dukes B & 9 \\
Dukes C & 1 \\
Dukes D & 5 \\
\hline
\end{tabular}

these patients had a family history of CRC. The numbers of patients having different stages of cancer according to Dukes classification are shown in Table 2.

3.3. Diagnostic Accuracy of Fecal Markers in Adenomas and CRCs. $\mathrm{M}_{2} \mathrm{PK}$ was positive in $32.5 \%$ of the patients with normal colonoscopy, in $43.7 \%$ with adenomas sized $<1 \mathrm{~cm}$, in $60 \%$ with adenomas sized $\geq 1 \mathrm{~cm}$, and in $94.7 \%$ with CRCs. $\mathrm{M}_{2} \mathrm{PK}$ sensitivity for adenomas sized $>1 \mathrm{~cm}$ was $60 \%$, and specificity was $67.5 \%$. Sensitivity and specificity for CRC were $94.7 \%$ and $67.5 \%$. Sensitivity and specificity for iFOBT for adenomas sized $\geq 1 \mathrm{~cm}$ were $80 \%$ and $72.5 \%$ and for CRC were 94.7\% and $72.5 \%$. The $\mathrm{Hb} / \mathrm{Hp}$ ( $\mathrm{Hb}$ and $\mathrm{Hb} / \mathrm{Hp}$ ColonView Biohit test) complex was positive in $47.1 \%$ of the patients with normal colonoscopy, in 50\% with hyperplastic polyps, in $54 \%$ with adenomas sized $<1 \mathrm{~cm}$, in $80 \%$ with adenomas sized $\geq 1 \mathrm{~cm}$, and in $100 \%$ with CRC. Sensitivity and specificity of $\mathrm{Hb} / \mathrm{Hp}$ complex for adenomas sized $\geq 1 \mathrm{~cm}$ were $80 \%$ and $52.9 \%$ and for CRC were $100 \%$ and $52.9 \%$.

FC and MMP-9 differed significantly between the control and CRC group $(p=0.022 ; p<0.001)$; however, no difference was found in FC and MMP-9 concentrations between the control and the adenoma groups. FC was significantly lower in adenomas sized $<1 \mathrm{~cm}$ compared to CRCs but did not differ when compared to adenomas sized $\geq 1 \mathrm{~cm}$ with CRCs 
TABLE 3: Sensitivities, specificities, and positive and negative predictive values of the fecal markers.

\begin{tabular}{|c|c|c|c|c|c|}
\hline Parameters & $\mathrm{M}_{2}-\mathrm{PK}_{\text {ScheBo }}$ & $\mathrm{HB}_{\text {SchBo }}$ & $\mathrm{HB} / \mathrm{HP}_{\text {biohit }}$ & Calprotectin & MMP-9 \\
\hline \multicolumn{6}{|l|}{ Sensitivity } \\
\hline Adenoma sized $\geq 1 \mathrm{~cm}$ & 60 & 80 & 80.0 & & \\
\hline $\mathrm{CRC}$ & 94.7 & 94.7 & 100.0 & 77.8 & 72.2 \\
\hline Adenoma sized $\geq 1 \mathrm{~cm}+\mathrm{CRC}$ & 76.9 & 87.2 & 90.9 & & \\
\hline \multicolumn{6}{|l|}{ Specificity } \\
\hline Adenoma sized $\geq 1 \mathrm{~cm}$ & 67.5 & 72.5 & 52.9 & & \\
\hline CRC & 67.5 & 72.5 & 52.9 & 70.0 & 95.0 \\
\hline Adenoma sized $\geq 1 \mathrm{~cm}+\mathrm{CRC}$ & 67.5 & 72.5 & 52.9 & & \\
\hline \multicolumn{6}{|l|}{$P P V(\%)$} \\
\hline Adenoma sized $\geq 1 \mathrm{~cm}$ & 80 & 59.2 & 42.9 & & \\
\hline CRC & 85.7 & 62 & 52.9 & 53.8 & 86.6 \\
\hline Adenoma sized $\geq 1 \mathrm{~cm}+$ CRC & 69.7 & 75.5 & 65.2 & & \\
\hline \multicolumn{6}{|l|}{$N P V(\%)$} \\
\hline Adenoma sized $\geq 1 \mathrm{~cm}$ & 77.1 & 96.6 & 85.7 & & \\
\hline CRC & 96.4 & 96.6 & 100.0 & 87.5 & 88.3 \\
\hline Adenoma sized $\geq 1 \mathrm{~cm}+\mathrm{CRC}$ & 75 & 85.3 & 85.7 & & \\
\hline
\end{tabular}

( $p=0.022, p=0.089$ ). MMP-9 proved to be significantly lower compared to either adenomas sized $<1 \mathrm{~cm}$ with CRCs or adenomas sized $\geq 1 \mathrm{~cm}$ with CRCs $(p \leq 0.001$ and $p \leq 0.001)$.

Sensitivity of FC for CRC was $77.8 \%$, while specificity for CRC was 70\%. The cut-off value of FC for the detection of CRC was $128.5 \mu \mathrm{g} / \mathrm{g}(\mathrm{AUC}=0.77, p=0.001)$. Sensitivity of MMP-9 for CRC was $72.2 \%$, while specificity was $95 \%$. The cut-off value of MMP-9 for the detection of CRC was $1.12 \mathrm{ng} / \mathrm{g}$ $(\mathrm{AUC}=0.77, p<0.001)$.

Using combinations of fecal markers, the highest sensitivity for detection of adenomas sized $\geq 1 \mathrm{~cm}$ was revealed when combining $\mathrm{M}_{2} \mathrm{PK}$, iFOBT, and FC (with the cut-off of $128.5 \mu \mathrm{g} / \mathrm{g}$ ) resulting in a sensitivity and specificity of $95 \%$ and $47.5 \%$ for the detection of adenomas sized $\geq 1 \mathrm{~cm}$.

Sensitivities, specificities, and positive and negative predictive values of the fecal markers are summarized in Table 3.

We did not find any relationship between platelet aggregation inhibitor therapy and positive results of the different hemoglobin tests (logistic regression: $\mathrm{Hb}_{\mathrm{ScheB}} p=0.4$; $\left.\mathrm{Hb} / \mathrm{Hp}_{\text {Biohit }} p=0.609\right)$.

\section{Discussion}

CRC is a major health problem worldwide. Despite being a good candidate for screening due to its detectable premalignant lesions, mortality rates of CRC are still significant in Hungary [17]. Early detection by an accurate, noninvasive, cost-effective, simple-to-use screening technique is central to decrease the incidence and mortality of this disease. Patient discomfort, invasiveness, embarrassment, high cost, and considerable expertise and equipment required for the procedure may all limit the appeal of this screening technique and the increasing number of examinations puts a huge burden on the gastroenterologists. Thus, there is still an unmet need for suitable noninvasive biomarkers to screen for CRC.
In this prospective colonoscopy-controlled study, we assessed the sensitivity, specificity, and positive and negative predictive values of different noninvasive fecal markers for the detection of adenomas and CRC. For adenomas sized $\geq 1 \mathrm{~cm}$, iFOBT showed the highest sensitivity and $\mathrm{M}_{2} \mathrm{PK}$ the highest specificity. For $\mathrm{CRC}, \mathrm{M}_{2} \mathrm{PK}$ and $\mathrm{Hb} / \mathrm{Hp}$ complex showed the highest sensitivity and fecal MMP-9 the highest specificity. FC and fecal MMP-9 concentrations did not differ between the control and the adenoma group, although they proved to be beneficial mainly in the detection of adenomas sized $\geq 1 \mathrm{~cm}$ and CRC. In CRCs, the sensitivities of FC and MMP- 9 were $78 \%$ and $72 \%$, with specificities of $70 \%$ and $95 \%$. The combination of $\mathrm{M}_{2} \mathrm{PK}$, iFOBT, and FC increased their sensitivity for the detection of adenomas sized $\geq 1 \mathrm{~cm}$ up to $95 \%$.

The study has some limitations. First, we collected stool samples before performing colonoscopy; thus, we were blinded to the findings and the number of high-grade adenomas finally proved to be low. We do not know whether there would be associations between adenomas and fecal markers if the number of adenomas with high-grade dysplasia would be higher. Second, $\mathrm{M}_{2} \mathrm{PK}$ and $\mathrm{Hb}$ tests and the $\mathrm{Hb} / \mathrm{Hp}$ complex were all qualitative tests based on a chromatographic method interpreted visually which may limit their assessment in case of borderline results. Therefore, it may be difficult to compare the results with those of FC and MMP-9. However, these tests are simple, do not require specific laboratory equipment, and therefore are less expensive than the quantitative methods.

The guaiac-based FOBT (gFOBT) is the oldest and most commonly used noninvasive test for detecting CRC [18, 19]. Although the test is relatively inexpensive and easy to perform, false-positive and false-negative results compose its main limitation resulting in limited sensitivity for detecting cancer and advanced adenomas [20]. The Hb/Hp complex shows higher stability against degradation than $\mathrm{Hb}$ itself. Sieg 
et al. revealed that $\mathrm{Hb} / \mathrm{Hp}$ complex has a comparable sensitivity to fecal $\mathrm{Hb}$ for CRCs (87\% for both) and higher sensitivity for adenomas (76\% versus 54\%) [4]. However, these tests are based on the bleeding property of the adenomas. Since earlystage cancers or advanced adenomas are unlikely to bleed continuously, $100 \%$ of clinical sensitivity cannot be achieved with the use of these tests. That is why the identification of novel fecal-based biomarkers is important.

$\mathrm{M}_{2} \mathrm{PK}$ is expressed by proliferating cells, in particular the tumor cells being direct target of several oncoproteins. Among the first studies assessing the sensitivity of $\mathrm{M}_{2} \mathrm{PK}$ for the detection of CRC, Shastri et al. revealed that fecal $\mathrm{M}_{2} \mathrm{PK}$ assay had sensitivity and specificity of 81.1 and $71.1 \%$ for diagnosing CRC at a cut-off value of $4 \mathrm{U} / \mathrm{mL}$ whereas FOBT showed a sensitivity of $36.5 \%$ and specificity of $92.2 \%$ for CRC. They concluded that $\mathrm{M}_{2} \mathrm{PK}$ is a poor screening biomarker, due to its low specificity [21]. However, a metaanalysis including 17 studies performed between 2006 and 2010 found the mean fecal $\mathrm{M}_{2}$ PK sensitivity and specificity to be $80.3 \%$ and $95.2 \%$ for CRC and a sensitivity of $44 \%$ for adenomas $>1 \mathrm{~cm}$ [22].

According to our results, $\mathrm{M}_{2} \mathrm{PK}, \mathrm{Hb}$, and $\mathrm{Hb} / \mathrm{Hp}$ tests show better sensitivity in the detection of CRC than advanced adenomas. The study by Kim et al. revealed that the sensitivity of $\mathrm{iM}_{2} \mathrm{PK}$, an immunochromatographic qualitative method for fecal $\mathrm{M}_{2} \mathrm{PK}$ for CRC, was $92.8 \%$ and for adenomatous lesions the sensitivity was $69.4 \%$ [23]. Compared with $\mathrm{M}_{2} \mathrm{PK}$ ELISA, $\mathrm{iM}_{2} \mathrm{PK}$ exhibited significantly enhanced sensitivity for CRC (97.5\% versus $80 \%, p=0.03$ ).

FC is valuable in differentiating functional and organic bowel diseases. FC was shown to be more sensitive (79\%) but less specific (72\%) for CRC and adenomatous polyps as a combined group than gFOBT [24]. MMP-9 is an important member of the gelatinases involved in the development of several human malignancies [25]. Yang et al. found that MMP-9 expression in colon cancer tissues was significantly higher than that in corresponding distal normal mucosa tissue [15]. However, the sensitivity of MMP-9 detected in feces has not been examined previously. Our results revealed a moderate sensitivity of $72 \%$ and a good specificity of $95 \%$ for fecal MMP-9 in CRC. However, neither FC nor fecal MMP-9 provided valuable information on the detection of adenomas.

In this study, we compared the sensitivity and specificity of several fecal markers for the detection of colorectal cancers. The strengths of this study are the design that allowed directly calculating sensitivity and specificity of the different fecal markers, since every patient underwent colonoscopy after stool sample collection. This was the first time when five biomarkers were simultaneously studied. Fecal $\mathrm{M}_{2} \mathrm{PK}$ has the advantage that it detects both bleeding and nonbleeding tumors and adenoma. Conversely, fecal $\mathrm{M}_{2} \mathrm{PK}$ does not have false-positive results due to various noncancerous sources of bleeding. Furthermore, FC, MMP-9, and fecal $\mathrm{M}_{2} \mathrm{PK}$ are also sensitive to intestinal inflammation (inflammatory bowel disease, diverticulitis) increasing the proportion of falsepositive cases. In this study, we performed examinations for patients with GI symptom(s) not as a part of screening process because by this method we could disclose falsepositive results and could determine specificity data as well.
In our cohort, the highest sensitivity and specificity were achieved by the use of combined $\mathrm{M}_{2} \mathrm{PK}$ and iFOBT test in the detection of CRC. FC seems to be a useful adjuvant to the investigation of patients at high risk for colorectal neoplasia, while fecal MMP-9 may be a promising factor for detection of CRC. Although, in CRC, sensitivity of $\mathrm{M}_{2} \mathrm{PK}$, iFOBT, and $\mathrm{Hb} / \mathrm{Hp}$ complex proved to be high, in adenomas sized $\geq 1 \mathrm{~cm}$, sensitivity decreased significantly. Therefore, none of these markers are unique for detection of precancerous lesions of the colorectum. However, our result revealed that combined use of $\mathrm{M}_{2} \mathrm{PK}$, iFOBT, and FC may be valuable in the detection of large adenomas.

We recommend these noninvasive fecal tests in low-risk patients and in patients who do not have comorbidities. Results of FOBT may be false positive if the source of bleeding is not an adenoma or a malignant disease (diverticulitis, hemorrhoids, and anticoagulant therapy). However, inflammatory diseases of the colon (diverticulitis, different infections, and inflammatory bowel diseases) and extraintestinal cancer (cancer in the hepatobiliary tract, pancreas) or inflammation (hepatitis) may affect the results of the inflammatory marker test; thus, in these cases, we recommend colonoscopy as a one-step investigation. High-risk patients (who had at least one relative with early CRC or adenoma or had at least two relatives with CRC or adenoma) with symptoms or patients who have early (under the age of 60) CRC or adenoma among their relatives should also undergo colonoscopy. However, it is not questionable whether continued efforts are needed to discover effective tests to identify patients with nonhereditary risk factors and to develop invasive and costeffective screening modalities.

\section{Competing Interests}

The authors declare that they have no competing interests.

\section{Authors' Contributions}

Klaudia Farkas and Tamás Molnár contributed equally to this study.

\section{Acknowledgments}

This paper was supported by the János Bolyai Research Scholarship of the Hungarian Academy of Sciences (BO/ 00632/14/5). 2 in 1 Quick Test $\left(\mathrm{M}_{2} \mathrm{PK}+\mathrm{Hb}\right.$ test $)$ was provided by ScheBo Biotech, Hungary. Biohit ColonView Hb/Hp test was provided by HUN-MED Kft.

\section{References}

[1] J. Ferlay, E. Steliarova-Foucher, J. Lortet-Tieulent et al., "Cancer incidence and mortality patterns in Europe: estimates for 40 countries in 2012," European Journal of Cancer, vol. 49, no. 6, pp. 1374-1403, 2013.

[2] The Hungarian Central Statistical Office, Demographic Yearbook, 1963-2012, KSH, Budapest, Hungry, 2013.

[3] F. Arvelo, F. Sojo, and C. Cotte, "Biology of colorectal cancer," Ecancermedicalscience, vol. 9, article 520, 2015. 
[4] A. Sieg, C. Thoms, K. Lüthgens, M. R. John, and H. SchmidtGayk, "Detection of colorectal neoplasms by the highly sensitive hemoglobin-haptoglobin complex in feces," International Journal of Colorectal Disease, vol. 14, no. 6, pp. 267-271, 1999.

[5] S. Vasilyev, E. Smirnova, D. Popov et al., "A new-generation fecal immunochemical test (FIT) is superior to quaiac-based test in detecting colorectal neoplasia among colonoscopy referral patients," Anticancer Research, vol. 35, no. 5, pp. 2873-2880, 2015.

[6] K. Lüthgens, A. Maier, I. Kampert, A. Sieg, and H. SchmidtGayk, "Hemoglobin-haptoglobin-complex: a highly sensitive assay for the detection of fecal occult blood," Clinical Laboratory, vol. 44, no. 7-8, pp. 543-551, 1998.

[7] M. Tamada, M. Suematsu, and H. Saya, "Pyruvate kinase M2: multiple faces for conferring benefits on cancer cells," Clinical Cancer Research, vol. 18, no. 20, pp. 5554-5561, 2012.

[8] H. R. Christofk, M. G. Vander Heiden, M. H. Harris et al., “The M2 splice isoform of pyruvate kinase is important for cancer metabolism and tumour growth," Nature, vol. 452, no. 7184, pp. 230-233, 2008.

[9] E. Eigenbrodt, D. Basenau, S. Holthusen, S. Mazurek, and G. Fischer, "Quantification of tumor type M2 pyruvate kinase (Tu M2-PK) in human carcinomas," Anticancer Research, vol. 17, no. 4, pp. 3153-3156, 1997.

[10] A. Poullis, R. Foster, M. A. Mendall, and M. K. Fagerhol, "Emerging role of calprotectin in gastroenterology," Journal of Gastroenterology and Hepatology, vol. 18, no. 7, pp. 756-762, 2003.

[11] J. Tibble, K. Teahon, B. Thjodleifsson et al., "A simple method for assessing intestinal inflammation in Crohn's disease," Gut, vol. 47, no. 4, pp. 506-513, 2000.

[12] M. Wagner, C. G. B. Peterson, P. Ridefelt, P. Sangfelt, and M. Carlos, "Fecal markers of inflammation used as surrogate markers for treatment outcome in relapsing inflammatory bowel disease," World Journal of Gastroenterology, vol. 14, no. 36, pp. 5584-5589, 2008.

[13] J. A. Tibble, G. Sigthorsson, S. Bridger, M. K. Fagerhol, and I. Bjarnason, "Surrogate markers of intestinal inflammation are predictive of relapse in patients with inflammatory bowel disease," Gastroenterology, vol. 119, no. 1, pp. 15-22, 2000.

[14] B. Johne, O. Kronborg, H. I. Tøn, J. Kristinsson, and P. Fuglerud, "A new fecal calprotectin test for colorectal neoplasia: clinical results and comparison with previous method," Scandinavian Journal of Gastroenterology, vol. 36, no. 3, pp. 291-296, 2001.

[15] B. Yang, F. Tang, B. Zhang, Y. Zhao, J. Feng, and Z. Rao, "Matrix metalloproteinase-9 overexpression is closely related to poor prognosis in patients with colon cancer," World Journal of Surgical Oncology, vol. 12, no. 1, article 24, 2014.

[16] A. Annaházi, T. Molnár, K. Farkas et al., "Fecal MMP-9: a new noninvasive differential diagnostic and activity marker in ulcerative colitis," Inflammatory Bowel Diseases, vol. 19, no. 2, pp. 316-320, 2013.

[17] I. Boncz, V. Brodszky, M. Péntek et al., "The disease burden of colorectal cancer in Hungary," European Journal of Health Economics, vol. 10, no. 1, pp. S35-S40, 2010.

[18] O. Kronborg, C. Fenger, J. Olsen, O. D. Jørgensen, and O. Søndergaard, "Randomised study of screening for colorectal cancer with faecal-occult-blood test," The Lancet, vol. 348, no. 9040, pp. 1467-1471, 1996.

[19] J. Kewenter, H. Brevinge, B. Engarás, E. Haglind, and C. Ährén, "Results of screening, rescreening, and follow-up in a prospective randomized study for detection of colorectal cancer by fecal occult blood testing: results for 68,308 subjects," Scandinavian Journal of Gastroenterology, vol. 29, no. 5, pp. 468473, 1994.

[20] M. J. Duffy, L. G. M. Van Rossum, S. T. Van Turenhout et al., "Use of faecal markers in screening for colorectal neoplasia: a European group on tumor markers position paper," International Journal of Cancer, vol. 128, no. 1, pp. 3-11, 2011.

[21] Y. M. Shastri, M. Naumann, G. M. Oremek et al., "Prospective multicenter evaluation of fecal tumor pyruvate kinase type M2 (M2-PK) as a screening biomarker for colorectal ecoplasia," International Journal of Cancer, vol. 119, no. 11, pp. 2651-2656, 2006.

[22] C. Tonus, M. Sellinger, K. Koss, and G. Neupert, "Faecal pyruvate kinase isoenzyme type M2 for colorectal cancer screening: a meta-analysis," World Journal of Gastroenterology, vol. 18, no. 30, pp. 4004-4011, 2012.

[23] Y. C. Kim, J. H. Kim, D. Y. Cheung et al., "The usefulness of a novel screening kit for colorectal cancer using the immunochromatographic fecal tumor M2 pyruvate kinase test," Gut and Liver, vol. 9, no. 5, pp. 641-648, 2015.

[24] J. Tibble, G. Sigthorsson, R. Foster, R. Sherwood, M. Fagerhol, and I. Bjarnason, "Faecal calprotectin and faecal occult blood tests in the diagnosis of colorectal carcinoma and adenoma," Gut, vol. 49, no. 3, pp. 402-408, 2001.

[25] M. Groblewska, M. Siewko, B. Mroczko, and M. Szmitkowski, "The role of matrix metalloproteinases (MMPs) and their inhibitors (TIMPs) in the development of esophageal cancer," Folia Histochemica et Cytobiologica, vol. 50, no. 1, pp. 12-19, 2012. 


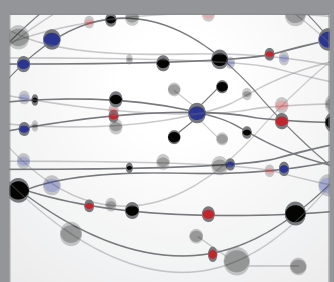

The Scientific World Journal
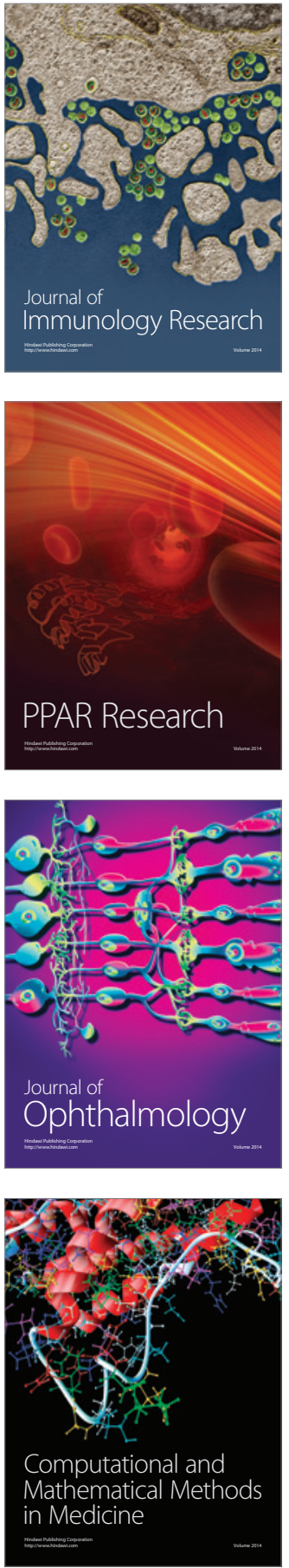

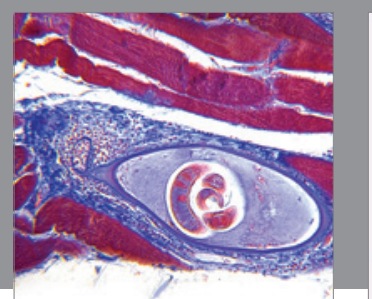

Gastroenterology Research and Practice

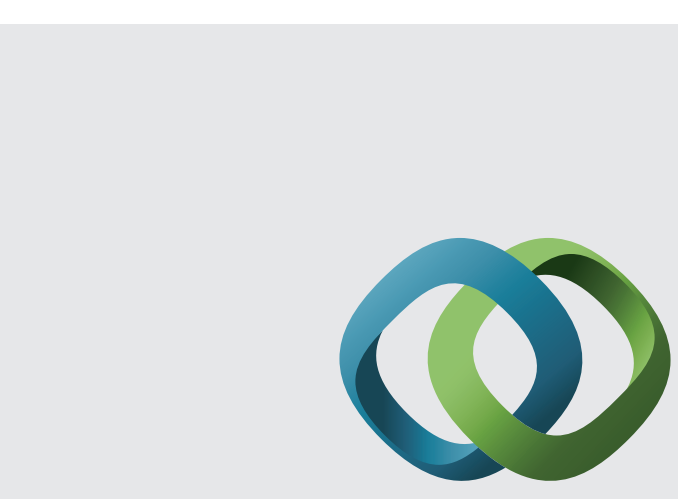

\section{Hindawi}

Submit your manuscripts at

http://www.hindawi.com
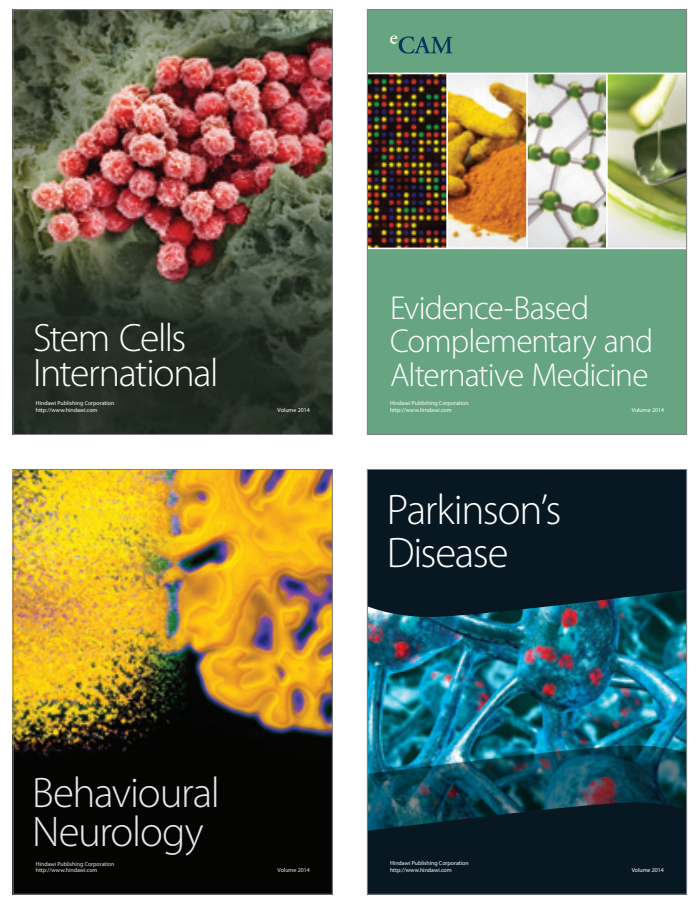
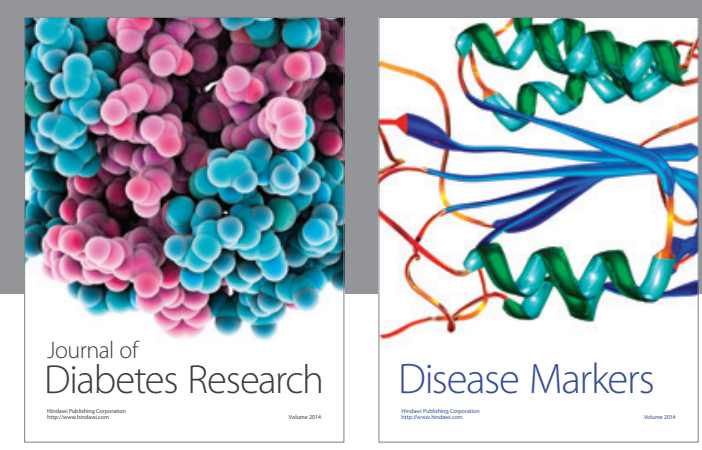

Disease Markers
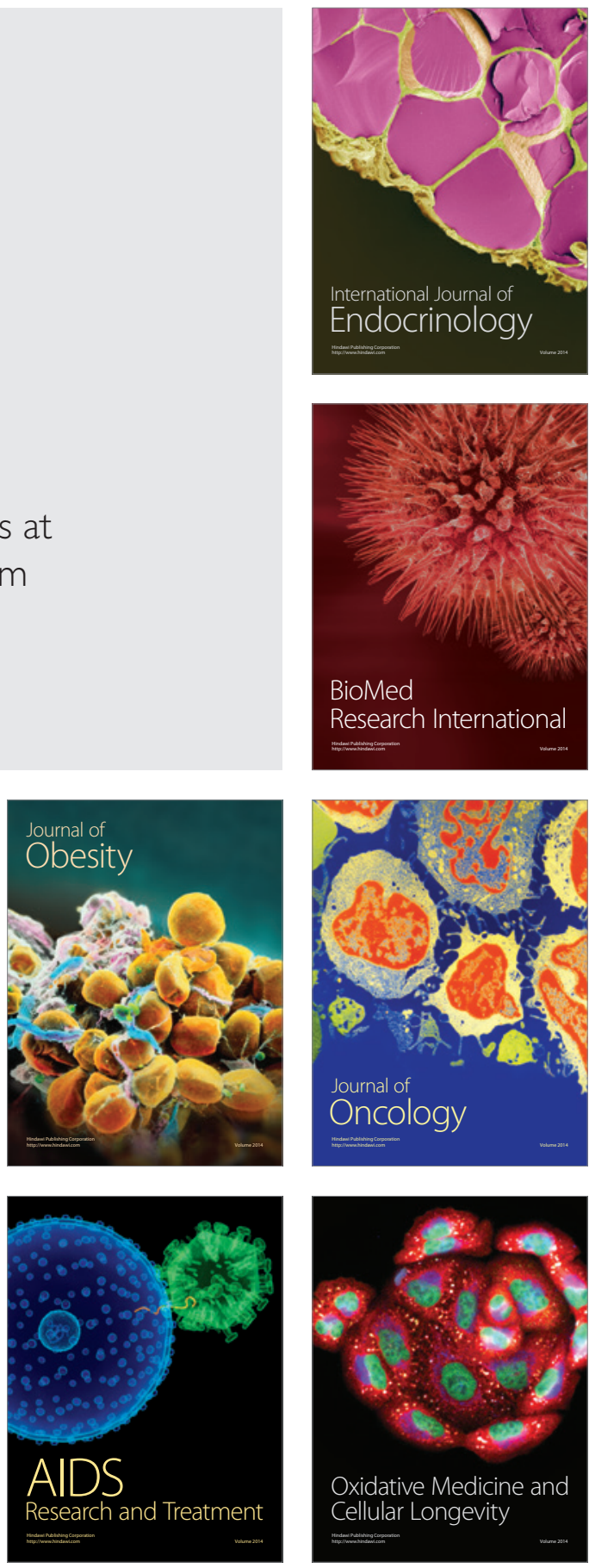